\title{
On the Absence of Spontaneous Symmetry Breaking and of Crystalline Ordering in Two-Dimensional Systems
}

\author{
Jürg Fröhlich ${ }^{1}$ and Charles Pfister ${ }^{2}$ \\ 1 Institut des Hautes Etudes Scientifiques, 35, route de Chartres, F-91440 Bures-sur-Yvette, France \\ 2 Département de Mathématiques, Ecole Polytechnique Fédérale, 61, av. de Cour, CH-1007 Lausanne, \\ Switzerland
}

\begin{abstract}
We develop a unified approach, based on Araki's relative entropy concept, to proving absence of spontaneous breaking of continuous, internal symmetries and translation invariance in two-dimensional statisticalmechanical systems. More precisely, we show that, under rather general assumptions on the interactions, all equilibrium states of a two-dimensional system have all the symmetries, compact internal and spatial, of the dynamics, except possibly rotation invariance. (Rotation invariance remains unbroken if connected correlations decay more rapidly than the inverse square distance.) We also prove that two-dimensional systems with a non-compact internal symmetry group, like anharmonic crystals, typically do not have Gibbs states.
\end{abstract}

\section{Introduction and Main Results}

It is well known that continuous symmetries of two-dimensional statistical mechanical systems or two-space-time-dimensional quantum field theories cannot, in general, be broken spontaneously (except in systems with interactions of very long range). Mathematical proofs of this fact have been known for quite a long time: They have appeared in work of Mermin and Wagner [1] concerning quantum spin systems on a two-dimensional lattice, of Mermin [2] concerning classical lattice spin systems, and in [3] where classical particle systems have been analyzed. For related results concerning quantum field theory, see [4], [5]. In [1] and [2] it is shown that the spontaneous magnetization vanishes and in [3] that the density of particles is constant, thus excluding the existence of crystalline order. Physical background material as well as the mathematical outline of the proofs are very well explained in [6]. The basic tool is Bogoliubov's inequality, which was used for the first time in this context by Hohenberg in his study of the Bose gas [7]. (A rigorous proof was later published in [8].) Using Bogoliubov's inequality, Fisher and Jasnow [9] proved clustering properties of the two-point function and, consequently, that the order parameter vanishes. McBryan and Spencer obtained a better decay for the two- 
point function of classical spin systems, using new techniques $[10]^{1}$. Shlosman generalized their work to the case of a compact connected Lie group [11]. (The extension of [2] to this general situation was given by Vuillermot and Romerio [12].)

In the current context of statistical mechanics, Garrison, Wong and Morisson $[13]^{2}$ were the first to prove a result about the invariance of the states of the system. Their argument employs Bogoliubov's inequality and the algebraic approach to statistical mechanics. They discussed the internal and the spatial symmetries of the system. Using a rather different approach, Dobrushin and Shlosman [14], and later Shlosman [15], proved that all equilibrium states are $G$ invariant in the case of classical spin systems, where $G$ is a compact connected Lie group. In the context of relativistic quantum field theory this result was anticipated (somewhat implicitly) in [4]. Recently the results in [14] were rederived and generalized in [16]. Since the new proof appearing in [16] is simpler technically, it turned out to be possible to obtain optimal results for some class of spin systems. At the same time, Simon and Sokal [17] proved some related as well as different results. They proposed a rigorous version of the entropy versus energy argument which captures one of the basic principles of statistical physics.

In this paper we present another approach to the problem, inspired by [16] and [18]. The basic physical idea can already be found in [19]. In Sect. 2, we show that (tempered) Gibbs states of particle systems are translation-invariant for a large class of potentials. In Sect. 3, we prove that all KMS-states of a quantum spin system are $G$-invariant, where $G$ is as above. This is in particular the case for the Heisenberg model. Our method can be used to extend the results of [16] to lattice systems of genuinely unbounded spins in two dimensions, when the internal symmetry group is a compact connected Lie group $G$. On the other hand, if the internal symmetry group $G$ is a noncompact connected Lie group, as in the case of the harmonic crystal, it is impossible to construct Gibbs states. Dobrushin and Shlosman proved such results in [20], and in Sect. 4, we derive similar results for a larger class of spin systems. Let us mention the interesting paper [21] of Jona-Lasinio, Pierini and Vulpiani, where this problem is discussed. We also derive results similar to those obtained by Brascamp, Lieb and Lebowitz in [22]. They used Bogoliubov's inequality to prove the divergence of some moments of the spins in the thermodynamic limit.

Throughout this paper, our main method is the same. We therefore do not repeat all steps for each case in detail. Our proof of Theorem 1 which establishes translation invariance of the Gibbs state in a class of classical particle systems is the most complete one.

Our basic strategy is the following: we make use of the fact that twodimensional systems support large fluctuations of finite energy. For example, it is possible to rotate all spins by a fixed amount on an arbitrarily large area without paying more than a finite (actually arbitrarily small) amount of energy independent of the area. It suffices to allow for a large transition region on which the spins are "rotated smoothly", i.e. on which the amount by which the spins are rotated decreases from a constant to the identity as the outer boundary of the transition

1 Their arguments can be extended to quantum spin systems

2 We thank J. Bricmont for pointing out this reference 
region is approached. It is at this point where the continuous character of the symmetry group enters. This is the basic physics.

In more mathematical terms, starting from a Gibbs state $P$, of some system with a continuous symmetry group, e.g. some spin system, we are able to construct perturbed states, $P_{n}, n=1,2,3, \ldots$, which are obtained from $P$ by rotating spins in a region of diameter $\propto n$ and satisfy the following two requirements:

i) The relative entropy, $S\left(P_{n} \mid P\right)$ of $P_{n}$ with respect to $P$ is defined by

$$
S\left(P_{n} \mid P\right)=\beta\left\langle H_{n}-H\right\rangle_{P}, \quad \beta=(k T)^{-1},
$$

i.e. it is proportional to the expected value in the state $P$ of the difference between the perturbed and the original Hamilton function. The requirement is that $S\left(P_{n} \mid P\right)$ be bounded uniformly in $n$. A simple application of Jensen's inequality (in the case of classical systems) then shows that $P$ and any limit of $\left(P_{n}\right)$ cannot be mutually singular ("orthogonal").

ii) All spins in a region, $\Lambda_{n}$, say a disk of radius $n$ centered at the origin, have been turned upside down. Restricted to $\Lambda_{n}, P_{n}$ coincides with $\hat{P}$, the Gibbs state obtained by turning all spins upside down.

The conclusion is that, because of $\mathrm{i}$ ), the relative entropy of $\hat{P}$ with respect to $P$ is finite, and this implies, as remarked, that $P=\hat{P}$, (provided $P$ is an extremal state, i.e. a pure phase).

The use of relative entropy as a means of comparing different Gibbs states of some system was pioneered by Araki who applied it to prove uniqueness of KMS states in one-dimensional quantum spin systems [18]. Our use of that concept provides a unified treatment of problems related to uniqueness of equilibrium states and absence of symmetry breaking. The method is not restricted to systems with a continuous symmetry group, contrary to the approach based on Bogoliubov's inequality.

\section{Two-Dimensional Systems of Classical Particles.}

2.1. Notations, Basic Concepts. We consider two-dimensional systems of classical particles in $X=\mathbb{R}^{2}$. The configuration space is defined as the set $\Omega$ of all finite or countable subsets, $\omega$, of $X$ such that $\omega \cap V$ is finite, for any bounded subset $V$ of $X$. Alternatively, we may define $\Omega$ as the set of all Radon measures of the form

$$
\sum_{x \in \omega} \varepsilon_{x}
$$

where $\omega$ is as above and $\varepsilon_{x}$ is the Dirac measure at $x$. We shall use both interpretations of $\Omega$. We thus have the two equivalent notations

$$
\sum_{x \in \omega} f(x) \text { and } \int \omega(d x) f(x)
$$

We use the shorthand $\omega_{\Lambda}$ for $\omega \cap \Lambda, \Lambda$ a subset of $X$ and write $\omega \mu$ instead of $\omega \cup \mu$, $\omega$ and $\mu \in \Omega$. The Lebesgue measure on $X$ is denoted by $\lambda$ and $\bar{\Lambda}=X \backslash \Lambda$. 
For any bounded subset, $\Lambda$, we introduce the counting variable

$$
N(\Lambda)(\omega) \equiv \omega(\Lambda)=|\omega \cap \Lambda| .
$$

For any Borel subset $V$, let

$$
\mathbf{F}(V)=\sigma\{N(\Lambda): \Lambda \subset V, \Lambda \text { bounded }\}
$$

be the $\sigma$-algebra of all events in $V$ generated by the random variables $N(\Lambda)$. Let $\mathbf{F}$ $=\mathbf{F}(X)$. There is exactly one probability measure on $(\Omega, \mathbf{F})$, namely the Poisson point process $\pi$ with intensity measure $z \cdot \lambda$, such that, for arbitrary, pairwise disjoint, bounded sets $\Lambda_{1}, \ldots, \Lambda_{n}$, the random variables $N\left(\Lambda_{1}\right), \ldots, N\left(\Lambda_{n}\right)$ are independent and have expected values $z \lambda\left(\Lambda_{1}\right), \ldots, z \lambda\left(\Lambda_{n}\right)$. Here $z$ is the activity of the particles. Thus $\pi$ describes an ideal gas of particles or a gas at inverse temperature $\beta=0$. If $V$ is a bounded subset and $f$ a nonnegative, $\mathbf{F}(V)$-measurable function we have the explicit formula

$$
\int \pi(d \omega) f(\omega)=e^{-z \lambda(V)} \sum_{n \geqq 0} \frac{z^{n}}{n !} \int_{(V)^{n}} \lambda\left(d x_{1}\right) \ldots \lambda\left(d x_{n}\right) f\left(\left\{x_{1}, \ldots, x_{n}\right\}\right) .
$$

We now assume that, at finite temperature, the particles interact via a two-body translation-invariant potential. The potential energy of a configuration $\alpha=\{x, y\}$ consisting of a particle at $x$ and a particle at $y$ is given by

$$
\phi(\alpha)=\phi(x-y)=\phi(y-x)
$$

Assumption A. The function $\phi: \mathbb{R}^{2} \rightarrow \mathbb{R}$ is bounded below and

a) $\phi$ is stable: there exists a positive number $B$ such that for any finite configuration $\omega$ (i.e. $\omega(X)<\infty)$

$$
\sum_{\alpha \subset \omega} \phi(\alpha) \geqq-B \omega(X)
$$

b) $\phi$ is regular: there exists a positive number $d$, and a positive monotone decreasing function, $\varphi$, on $\mathbb{R}^{+}$such that

$$
|\phi(x)| \leqq \varphi(|x|), \quad|x| \geqq d,
$$

and

$$
\int_{0}^{\infty} t \varphi(t) d t<\infty
$$

Let $\Lambda$ be any bounded subset of $X$. The energy of a configuration, $\eta$, of particles in $\Lambda$ (i.e. $\eta \subset \Lambda$ ), given some boundary condition $\omega \in \Omega$, is formally

$$
H_{\Lambda}(\eta \mid \omega)=\sum_{\substack{\alpha \subset \eta \omega_{\bar{A}} \\ \alpha \cap \eta \neq \emptyset}} \phi(\alpha)
$$

In particular, for each $x \in X$ we put

$$
H(x \mid \omega)=H_{x}\left(\varepsilon_{x} \mid \omega\right),
$$


which is the energy of a particle at $x$ given the configuration $\omega$. (The particle at $x$ may or may not belong to $\omega$.) We define the Gibbs distribution of a system confined to $\Lambda$, given some boundary condition $\omega$, as the probability measure on $(\Omega, \mathbf{F}(\Lambda))$ whose Radon-Nikodym density with respect to the restriction of $\pi$ to $\mathbf{F}(\Lambda)$ is

$$
P_{\Lambda}(\eta \mid \omega)=Z(\Lambda \mid \omega)^{-1} \exp \left(-\beta H_{\Lambda}(\eta \mid \omega)\right),
$$

where $Z(\Lambda \mid \omega)$ is a normalization factor (partition function):

$$
e^{-z \lambda(\Lambda)} \leq Z(\Lambda \mid \omega) \equiv \int \pi(d \eta) \exp \left(-\beta H_{\Lambda}(\eta \mid \omega)\right) .
$$

Formula (2.5) is meaningful whenever, for a given $\omega,(2.3)$ is defined for all $\eta \subset \Lambda$, and $Z(\Lambda \mid \omega)<\infty$. This is in particular the case for the class of configurations corresponding to the following subset $\Omega_{\infty} \subset \Omega$ : Let $\Lambda_{n}$ be the family of disks centered at the origin with radius $n, n \in \mathbb{N}$,

$$
\Lambda_{n}=\{x \in X:|x| \leqq n\} .
$$

Let

$$
\Omega_{N}=\left\{\omega \in \Omega: \omega\left(\Lambda_{n}\right) \leqq N \lambda\left(\Lambda_{n}\right), \quad \forall n \in \mathbb{N}\right\} .
$$

Then

$$
\Omega_{\infty}=\bigcup_{N \geqq 1} \Omega_{\mathrm{N}}
$$

Definition [23]. A Gibbs state $P$ is a probability measure on $(\Omega, \mathbf{F})$ such that, for all bounded sets $\Lambda,(2.5)$ is well-defined for $P$-almost all $\omega$, and the conditional probability of $P$ with respect to $\mathbf{F}(\bar{\Lambda})$ is given by the Gibbs distribution in $\Lambda$.

This section is largely inspired by Chapter 1 of [24] to which we refer the reader for additional information. See also [25].

2.2 The Main Result on Particle Systems. In order to state and prove our main result we need some additional assumptions on the potential $\phi$.

Assumption B. $\phi$ is a twice continuously differentiable function (except at $0 \in \mathbb{R}^{2}$ ).

Let $a \in \mathbb{R}^{2}$ be a unit vector, $|a|=1$. Let $0<\varepsilon<1$ be given and $t \in \mathbb{R}$. We define on $\mathbb{R}^{2}$

$$
\psi_{\varepsilon}(x)=\sup _{\substack{a: \\|a|=1}} \sup _{\substack{t: \\|t| \leqq \varepsilon|x|}}\left|\frac{d^{2}}{d t^{2}} \phi(x+t a)\right||x|^{2} .
$$

We may regard $\psi_{\varepsilon}$ as a potential and define

$$
\psi_{\varepsilon}(\alpha)=\psi_{\varepsilon}(x-y)
$$

for any configuration $\alpha=\{x, y\}$; see (2.2). Moreover we introduce, for $\omega \in \Omega$ [see (2.4)],

$$
H_{\psi_{\varepsilon}}(x \mid \omega)=\sum_{\substack{y \in \omega \\ y \neq x}} \psi_{\varepsilon}(x-y)
$$


Assumption C. There exists $0<\varepsilon<1$ such that $\psi_{\varepsilon}$, interpreted as a potential, is stable: $\sum_{\alpha \subset \omega} \psi_{\varepsilon}(\alpha) \geqq-\widetilde{B} \omega(X)$,

with $0<\widetilde{B}<\infty, \quad \omega \in \Omega$ and $\omega(X)<\infty$.

Let $a \in \mathbb{R}^{2}$. The translation $T_{a}$ on $X$ is defined as

$$
x \rightarrow T_{a} x=x+a .
$$

This transformation induces a transformation on $\Omega$, also denoted by $T_{a}$ :

with

$$
\omega \rightarrow T_{a} \omega,
$$

$$
T_{a} \omega(h)=\int \omega(d x) h(x+a),
$$

$h$ being a measurable function on $X$. Let $P$ be a Gibbs state. $T_{a} P$ is defined by $d\left(T_{a} P\right)(\omega)=d P\left(T_{a} \omega\right)$. We say that $P$ is translation invariant if $P=T_{a} P$, for all $a \in \mathbb{R}^{2}$

Theorem 1. Let $P$ be an extremal Gibbs state for a particle system on $\mathbb{R}^{2}$ with activity $z$, inverse temperature $\beta$ and with two-body potential satisfying assumptions $A, B$ and C. If

a) there exists a constant $K<\infty$ such that for any bounded subset $\Lambda$ of $\mathbb{R}^{2}$

$$
\int P(d \omega) N(\Lambda)(\omega) \leqq K \lambda(\Lambda),
$$

b) there exists a constant $C<\infty$ such that for all $x \in X$,

$$
\int P(d \omega) H_{\psi_{e}}(x \mid \omega) \exp (-\beta H(x \mid \omega)) \leqq C ;
$$

then $P$ is translation-invariant.

\section{Remarks.}

1) The assumption that $P$ be extremal is no loss of generality, because a Gibbs state satisfying a) and b) has a decomposition into extremal Gibbs states having the same properties almost surely.

2) This result can be extended to some systems consisting of several species of particles, e.g. ones with charge interacting via a smooth two-body potential.

In the corollary below we discuss a specific class of two-body potentials for which hypotheses a) and b) of Theorem 1 can be verified. Our result involves the notions of superstability and tempered Gibbs state for which we refer the reader to Ruelle's paper [25]. Let $\phi$ be some potential and let $\phi^{+}, \phi^{-}$denote the positive part, the negative part of $\phi$, respectively, so that $\phi=\phi^{+}-\phi^{-}$and $|\phi|=\phi^{+}+\phi^{-}$. We define $H_{|\phi|}(x \mid \omega)$ and $H_{\phi^{-}}(x \mid \omega)$ as in (2.4).

Corollary. Let $\phi$ be a superstable potential satisfying hypotheses $A, B$ and $C$ above. Assume, moreover, that

$$
H_{\psi_{\varepsilon}}(x \mid \omega) \exp \left(-\beta H_{|\phi|}(x \mid \omega)\right) \leqq C,
$$

for some finite constant $C$ independent of $x$ and $\omega$. Then all tempered Gibbs states are translation-invariant. 
Proof. Our proof is based on the work of Ruelle, [25]. Since one can always decompose a tempered Gibbs state into tempered extremal Gibbs states, it suffices to consider an extremal Gibbs state and to verify for it the hypotheses a) and b) of Theorem 1. Hypothesis a) follows from the temperedness of $P$, and hypothesis $\mathrm{b}$ ) is verified as follows:

$$
\begin{aligned}
H_{\psi_{e}}(x \mid \omega) \exp (-\beta \mathrm{H}(x \mid \omega)) & =H_{\psi_{e}}(x \mid \omega) \exp \left(-\beta H_{|\phi|}(x \mid \omega)\right) \exp \left(2 \beta H_{\phi^{-}}(x \mid \omega)\right) \\
& \leqq C \exp \left(2 \beta H_{\phi^{-}}(x \mid \omega)\right) .
\end{aligned}
$$

Thus we must prove that

$$
\int P(d \omega) \exp \left(2 \beta \mathrm{H}_{\phi^{-}}(x \mid \omega)\right)<\infty .
$$

This, however, is done in the proof of a), Proposition 5.2 of [25].

\section{Remarks}

1) The condition

$$
H_{\psi_{\varepsilon}}(x \mid \omega) \exp \left(-\beta H_{|\phi|}(x \mid \omega)\right) \leqq C
$$

is mild. It is satisfied by "most" potentials for which $A, B$ and $C$ hold. In particular, this is true for all potentials of Lennard-Jones type; see Proposition 1.4 in [25].

2) Our results can be extended to systems of several species of particles with interactions including many body potentials.

2.3 Proof of Theorem 1. It is sufficient to prove the theorem for $|a| \leqq 1$. Let $a$ be fixed and let $P_{a}=T_{a}^{-1} P$. We want to prove that $P_{a}$ and $P$ are equivalent by showing that for any $\mathbf{F}(\Lambda)$-measurable subsets $A \subset \Omega$ and $A^{c}=\Omega \backslash A, \Lambda$ bounded,

$$
0 \leqq-\left(P(A) \log \frac{P_{a}(A)}{P(A)}+P\left(A^{c}\right) \log \frac{P_{a}\left(A^{c}\right)}{P\left(A^{c}\right)}\right) \leqq K,
$$

and

$$
0 \leqq-\left(P_{a}(A) \log \frac{P(A)}{P_{a}(A)}+P_{a}\left(A^{c}\right) \log \frac{P\left(A^{c}\right)}{P_{a}\left(A^{c}\right)}\right) \leqq K,
$$

where $0<K<\infty$ is independent of $A, A^{c}$ and $\Lambda$. We obtain these inequalities by constructing a sequence of transformations $\left(T_{n}\right)$ on $X$ such that

$$
\begin{array}{ll}
T_{n} x=x+a=T_{a} x, & x \in \Lambda_{n} \\
T_{n} x=x \quad, & x \notin \Lambda_{p n},
\end{array}
$$

where $\Lambda_{n}=\{x \in X:|x| \leqq n\}, n \in \mathbb{N}$, and $0<p$ is suitably large. As in (2.8) we define a transformation $T_{n}$ on $\Omega$ inducing a transformation of measures on $(\Omega, \mathbf{F})$. We set

$$
P_{n}=T_{n}^{-1} P \text {. }
$$

We then show that the relative entropy

$$
0 \leqq S\left(P_{n} \mid P\right)=-\int P(d \omega) \log \frac{d P_{n}}{d P}(\omega) \leqq K
$$


is bounded by a constant $K$ independent of $n$. If $n$ is large enough so that $\Lambda_{n} \supset \Lambda$, then (2.9) is bounded by (2.11). This follows by using Jensen's inequality. The other inequality, (2.10), is proved in the same way.

Let $u$ be a non-negative, smooth, monotone decreasing function on $\mathbb{R}^{+}$with the properties

$$
u(x)=1, \quad x \leqq 1, \text { and } \quad u(x)=0, \quad x \geqq p,
$$

for some $p$, moreover

$$
\left|\frac{d u}{d x}\right| \leqq \varepsilon, \quad 0<\varepsilon<1
$$

We define

$$
T_{n}: x \rightarrow x+a \cdot u\left(\frac{|x|}{n}\right), \quad n \geqq 1 .
$$

$T_{n}$ is a smooth function from $X$ into $X$. It is one-to-one because

$$
|u(|x|)-u(|y|)| \leqq \varepsilon|| x|-| y|| \leqq \varepsilon|x-y| .
$$

Let $P_{n}=T_{n}^{-1} P$, as above.

Lemma 2.1. $P_{n}$ is absolutely continuous with respect to $P$. Let $\Lambda \equiv \Lambda_{p n}$ and $T \equiv T_{n}$. Then

$$
\frac{d P_{n}}{d P}(\omega)=\prod_{x \in \omega_{A}} J_{T}(x) \exp \beta\left(H_{\Lambda}\left(\omega_{\Lambda} \mid \omega\right)-H_{\Lambda}\left(T \omega_{\Lambda} \mid \omega\right)\right),
$$

where $J_{T}(x)$ is the Jacobian of the transformation $T$.

Proof. Let $V$ be a bounded subset, $V \supset \Lambda$. Let $h$ be $\mathbf{F}(V)$-measurable.

$$
\begin{aligned}
\int P_{n}(d \omega) h(\omega) & =\int P(d \omega) h\left(T^{-1} \omega\right) \\
& =\int P(d \omega) \frac{1}{Z(V \mid \omega)} \int \pi(d \eta) \exp \left(-\beta H_{V}(\eta \mid \omega)\right) h\left(T^{-1} \eta\right)
\end{aligned}
$$

because $P$ is a Gibbs state.

$$
\begin{aligned}
\int \pi(d \eta) \exp \left(-\beta H_{V}(\eta \mid \omega)\right) h\left(T^{-1} \eta\right) \\
=e^{-z \lambda(V)} \sum_{n \geqq 0} \frac{z^{n}}{n !} \int_{(V)^{n}} \lambda\left(d x_{1}\right) \ldots \lambda\left(d x_{n}\right) h\left(T^{-1} x_{1}, \ldots, T^{-1} x_{n}\right) \\
\quad \cdot \exp \left(-\beta H_{V}\left(\left\{x_{1}, \ldots, x_{n}\right\} \mid \omega\right)\right) .
\end{aligned}
$$

Changing variables, $x_{i} \rightarrow T x_{i}$, in (2.12) yields the following integrand on the right hand side of (2.12):

$$
h\left(x_{1}, \ldots, x_{n}\right) \prod_{i=1}^{n} J_{T}\left(x_{i}\right) \exp \left(-\beta H_{\mathrm{V}}\left(\left\{T x_{1}, \ldots, T x_{n}\right\} \mid \omega\right)\right) .
$$

We multiply and divide this last expression by

$$
\exp \left(-\beta H_{V}\left(\left\{x_{1}, \ldots, x_{n}\right\} \mid \omega\right)\right) \text {. }
$$


To complete the proof we notice that

$$
\prod_{i=1}^{n} J_{T}\left(x_{i}\right)=\prod_{\substack{\mathrm{i}: \\ x_{i} \in \Lambda}} J_{T}\left(x_{i}\right)
$$

and

$$
H_{V}\left(\omega_{V} \mid \omega\right)-H_{V}\left(T \omega_{V} \mid \omega\right)=H_{\Lambda}\left(\omega_{\Lambda} \mid \omega\right)-H_{\Lambda}\left(T \omega_{\Lambda} \mid \omega\right)
$$

Lemma 2.2. Let $T \equiv T_{n}$, and let $\hat{T}$ be the transformation defined by replacing a by $-a$ in the definition of $T_{n}$. Let $\hat{P}_{n}=\hat{T}^{-1} P$.

There exists a constant $0<K<\infty$ independent of $n$ such that

$$
S\left(P_{n} \mid P\right) \leqq K, \quad S\left(\hat{P}_{n} \mid P\right) \leqq K .
$$

Proof. We introduce a duplication of the system and consider the probability measures $P \otimes P$ and $P_{n} \otimes \hat{P}_{n}$ on $\Omega \times \Omega$. We compute

$$
\begin{aligned}
S\left(P_{n} \otimes \hat{P}_{n} \mid P \otimes P\right) & \equiv-\int P(d \omega) P\left(d \omega^{\prime}\right) \log \frac{d\left(P_{n} \otimes \hat{P}_{n}\right)}{d(P \otimes P)}\left(\omega, \omega^{\prime}\right) \\
& =S\left(P_{n} \mid P\right)+S\left(\hat{P}_{n} \mid P\right) .
\end{aligned}
$$

We first bound in (2.13) the terms

$$
\beta\left(H_{\Lambda}\left(\widehat{T} \omega_{\Lambda} \mid \omega\right)-H_{\Lambda}\left(\omega_{\Lambda} \mid \omega\right)+H_{\Lambda}\left(T \omega_{\Lambda} \mid \omega\right)-H_{\Lambda}\left(\omega_{\Lambda} \mid \omega\right)\right) .
$$

Let $\tilde{\Lambda}=\Lambda_{p n} \backslash \Lambda_{n}, \quad\left(\Lambda_{p n} \equiv \Lambda\right)$. We have

$$
H_{\Lambda}\left(T \omega_{\Lambda} \mid \omega\right)-H_{\Lambda}\left(\omega_{\Lambda} \mid \omega\right)=\sum_{\alpha \subset \omega: \alpha(\tilde{\Lambda})>0}(\phi(T \alpha)-\phi(\alpha)),
$$

with $\alpha(\tilde{\Lambda})>0$ indicating that $\alpha \cap \tilde{\Lambda} \neq \emptyset$.

Let $\alpha=\{x, y\}$ and let

$$
f(t)=\phi\left(x-y+a t\left(u\left(\frac{|x|}{n}\right)-u\left(\frac{|y|}{n}\right)\right)\right) .
$$

Using Taylor's formula for $|t| \leqq 1$, we get

$$
\phi(T \alpha)=f(1)=f(0)+f^{\prime}(0)+\frac{1}{2} f^{\prime \prime}(\theta),
$$

and

$$
\phi(\hat{T} \alpha)=f(-1)=f(0)-f^{\prime}(0)+\frac{1}{2} f^{\prime \prime}(\hat{\theta}),
$$

with $0 \leqq|\theta|,|\hat{\theta}| \leqq 1$. Since $f(0)=\phi(\alpha)$, we see that only the terms $f^{\prime \prime}(\theta)$ and $f^{\prime \prime}(\hat{\theta})$ contribute to (2.13). Since

$$
\left|u\left(\frac{|x|}{n}\right)-u\left(\frac{|y|}{n}\right)\right| \leqq \varepsilon \frac{1}{n}|x-y|,
$$

we have

$$
\left|f^{\prime \prime}(\theta)\right| \leqq \psi_{\varepsilon}(x-y)\left(\frac{\varepsilon}{n}\right)^{2}
$$


Let $\psi=\psi_{\varepsilon}$. Then

$$
\sum_{\substack{\alpha \subset \omega \\ \alpha(\tilde{\Lambda})>0}} \psi(\alpha)=\sum_{x \in \omega_{\tilde{\Lambda}}} H_{\psi}(x \mid \omega)-\sum_{\alpha \in \omega_{\tilde{\Lambda}}} \psi(\alpha)
$$

Therefore we obtain the following bound for the contribution of (2.14) to (2.13):

$$
\beta\left(\frac{\varepsilon}{n}\right)^{2} \int P(d \omega)\left(-\sum_{\alpha \in \omega_{\tilde{\Lambda}}} \psi(\alpha)\right)+\beta\left(\frac{\varepsilon}{n}\right)^{2} \int P(d \omega) \sum_{x \in \omega_{\tilde{\Lambda}}} H_{\psi}(x \mid \omega) .
$$

The first term in (2.15) is bounded by

$$
\beta \frac{\varepsilon^{2} \widetilde{B}}{n^{2}} \int P(d \omega) N(\tilde{A})(\omega) \leqq K^{\prime} \varepsilon^{2} \widetilde{B}
$$

for $\lambda(\tilde{\Lambda})=0\left(n^{2}\right)$, and $\psi$ is stable. The second term of (2.15) can be written as

$$
\beta \frac{\varepsilon^{2}}{n^{2}} \int_{\Omega} P(d \omega) \int_{X} \chi_{\tilde{\Lambda}}(x) H_{\psi}(x \mid \omega) \omega(d x),
$$

with $\chi_{\tilde{\Lambda}}(\cdot)$ the characteristic function of the set $\tilde{\Lambda}$. The proof is completed by using Lemma 2.3 [26]. If $P$ is a Gibbs state, $\Lambda$ a bounded subset, $z$ the activity, then

$$
\int_{\Omega} P(d \omega) \int_{X} \chi_{\Lambda}(x) H_{\psi}(x \mid \omega) \omega(d x)=z \int_{X} \lambda(d x) \chi_{\Lambda}(x) \int_{\Omega} H_{\psi}(x \mid \omega) e^{-\beta H(x \mid \omega)} P(d \omega) .
$$

Using this lemma we see that (2.16) is bounded above by a constant The contribution of the Jacobians is estimated in the same way as the first term in (2.15). Thus Lemma 2.2 is proved, and this implies the bounds (2.9), (2.10). Therefore $P$ and $P_{a}$ are equivalent. The proof of Theorem 1 is now completed by appealing to the following:

Lemma 2.4 [27]. If $P$ and $P_{a}$ are equivalent, and $P$ is extremal, then

$$
P=P_{a} \text {. }
$$

It remains to prove Lemma 2.3, which is a special case of Theorem 2 in [26]. Proof of Lemma 2.3.

$$
\begin{aligned}
& z \int_{X} \lambda\left(d x_{0}\right) \chi_{\Lambda}\left(x_{0}\right) \int_{\Omega} P(d \omega) H_{\psi}\left(x_{0} \mid \omega\right) e^{-\beta H\left(x_{0} \mid \omega\right)}=\int_{\Lambda} \lambda\left(d x_{0}\right) e^{-z \lambda(\Lambda)} \int_{\Omega} P(d \omega) \frac{1}{Z(\Lambda \mid \omega)} \\
& \cdot \sum_{n \geqq 0} \frac{z^{n+1}}{n !} \int_{(\Lambda)^{n}} \lambda\left(d x_{1}\right) \ldots \lambda\left(d x_{n}\right) H_{\psi}\left(x_{0} \mid x_{1}, \ldots, x_{n}, \omega_{\tilde{\Lambda}}\right) e^{-\beta H\left(x_{0} \mid x_{1}, \ldots, x_{n}, \omega_{\tilde{A}}\right)} e^{-\beta H_{\Lambda}\left(x_{1}, \ldots, x_{n} \mid \omega_{\tilde{\Lambda}}\right)} .
\end{aligned}
$$

Since $x_{0} \in \Lambda$,

$$
H\left(x_{0} \mid x_{1}, \ldots, x_{n}, \omega_{\bar{\Lambda}}\right)+H_{\Lambda}\left(x_{1}, \ldots, x_{n} \mid \omega_{\bar{\Lambda}}\right)=H_{\Lambda}\left(x_{0}, x_{1}, \ldots, x_{n} \mid \omega_{\bar{\Lambda}}\right) .
$$

Moreover,

$$
H_{\psi}\left(x_{0} \mid x_{0}, \mathrm{x}_{1}, \ldots, x_{n}, \omega_{\bar{\Lambda}}\right)=H_{\psi}\left(x_{0} \mid x_{1}, \ldots, x_{n}, \omega_{\bar{X}}\right) .
$$


Using (2.17) and (2.18)

$$
\begin{aligned}
\frac{1}{n !} \int_{\Lambda} \lambda\left(d x_{0}\right) \int_{(\Lambda)^{n}} \lambda\left(d x_{1}\right) \ldots \lambda\left(d x_{n}\right) H_{\psi}\left(x_{0} \mid x_{1}, \ldots, x_{n}, \omega_{\bar{\Lambda}}\right) \\
\quad \cdot \exp \left(-\beta H\left(x_{0} \mid x_{1}, \ldots, x_{n}, \omega_{\bar{\Lambda}}\right) \exp \left(-\beta H_{\Lambda}\left(x_{1}, \ldots, x_{n} \mid \omega_{\bar{\Lambda}}\right)\right.\right. \\
=(n+1) \frac{1}{(n+1) !} \int_{(\Lambda)^{n+1}} \lambda\left(d x_{0}\right) \ldots \lambda\left(d x_{n}\right) \exp \left(-\beta H_{\Lambda}\left(x_{0}, x_{1}, \ldots, x_{n} \mid \omega_{\bar{\Lambda}}\right)\right) \\
\quad \cdot H_{\psi}\left(x_{0} \mid x_{0}, x_{1}, \ldots, x_{n}, \omega_{\bar{\Lambda}}\right) \\
=\frac{1}{(n+1) !} \int_{(\Lambda)^{n+1}} \lambda\left(d x_{0}\right) \ldots \lambda\left(d x_{n}\right)\left(\sum_{x_{i} \in \Lambda} H_{\psi}\left(x_{i} \mid x_{0}, \ldots, x_{n}, \omega_{\bar{\Lambda}}\right)\right) \\
\quad \cdot \exp \left(-\beta H_{\Lambda}\left(x_{0}, \ldots, x_{n} \mid \omega_{\bar{\Lambda}}\right)\right) .
\end{aligned}
$$

Summing over $n$ and integrating over $\omega_{\bar{\Lambda}}$, we get

$$
\int_{\Omega} P(d \omega) \int_{X} \chi_{\Lambda}(x) H_{\psi}(x \mid \omega)(d x) .
$$

We close this section with:

A Remark Concerning the Breaking of Rotation Invariance. The methods developed in this section can also be used to study the absence of breaking of rotation invariance in classical particle systems with rotation-invariant, superstable twobody potentials, (with properties analogous to the ones required in Assumptions A through $\mathrm{C}$ above).

It turns out, however, that the breaking of rotation-invariance, i.e. directional ordering, is possible, in principle, in two-dimensional systems with connected correlations which do not fall off more rapidly than the inverse square distance (so that there is some divergent "susceptibility").

Let $A(\omega)$ and $B(\omega)$ be some bounded F-measurable functions of $\omega$. We define

$$
\langle A\rangle_{P} \equiv \int P(d \omega) A(\omega),
$$

and

$$
\langle A ; B\rangle_{P} \equiv\langle A \cdot B\rangle_{P}-\langle A\rangle_{P}\langle B\rangle_{P},
$$

where $P$ is some tempered Gibbs state of the system. Next, we define a spacedependent rotation, $T_{\alpha}$. Let $x=\left(\begin{array}{l}x_{1} \\ x_{2}\end{array}\right)$. Then

$$
T_{\underline{\alpha}}\left(\begin{array}{l}
x_{1} \\
x_{2}
\end{array}\right) \equiv\left(\begin{array}{r}
\cos \underset{\sim}{\alpha}(x) x_{1}+\sin \underset{\sim}{\alpha}(x) x_{2} \\
-\sin \underset{\sim}{\alpha}(x) x_{1}+\cos \underset{\sim}{\alpha}(x) x_{2}
\end{array}\right),
$$

where

$$
\alpha(x)^{\text {e.g. }} \alpha u\left(\frac{|x|}{n}\right), \quad \alpha>0
$$


is a smooth function vanishing outside $\Lambda_{p n}$. Let $P_{\alpha} \equiv T_{\alpha}$ be defined as above. Let $A$ be some $\mathbf{F}(V)$-measurable function of $\omega$, where $V$ is a compact subset of $\mathbb{R}^{2}$. We choose $n$ so large that $V \subset \Lambda_{n}$. By definition

$$
\int P_{\alpha}(d \omega) A(\omega) \equiv \int P(d \omega) A\left(T_{\alpha} \omega\right)
$$

We propose to show that

$$
\int P_{\alpha}(d \omega) A(\omega)=\int P(d \omega) A(\omega)
$$

as $n \rightarrow \infty$, provided certain connected correlations have sufficiently rapid decrease. Since $T_{\alpha}$ is a constant rotation, $T_{\alpha}$, on $V$ it suffices to show that

$$
\frac{d}{d \alpha} \int P_{\alpha}(d \omega) A(\omega) /_{\alpha=0}=0,
$$

as $n \rightarrow \infty$, and this will imply the rotation invariance of $\langle A\rangle_{P}$.

A straightforward variant of Lemma 2.1 permits us to find an explicit expression for $\frac{d P_{\alpha}}{d P}$ which allows us then to calculate $\frac{d}{d \alpha}\left(\frac{d P_{\alpha}}{d P}\right)$ explicitly. From that expression we infer that, under fairly obvious hypotheses on the gradient of the two-body potential, (2.19) holds if

$$
\begin{gathered}
\left\langle A ; \varrho_{x}\right\rangle_{P}<0\left(1 / d^{2}\right), \\
\left\langle A ; \varrho_{x} \varrho_{y}\right\rangle_{P}<0\left(1 / d^{2}\right),
\end{gathered}
$$

provided $\operatorname{dist}(x, V) \geqq d, \operatorname{dist}(y, V) \geqq d$. Here $\varrho_{x}(\omega) \equiv \omega_{\{x\}}$ is the "coordinate function." A typical choice of $A(\omega)$ would be, for example,

$$
A(\omega)=\varrho_{x_{1}} \varrho_{x_{2}}, \quad x_{1} \neq x_{2} .
$$

A related discussion of the absence of breaking of rotation (and translation) invariance, based on the BBGKY hierarchy, has recently appeared in very interesting papers by Gruber and Martin [28].

\section{Quantum Spin Systems}

3.1. Notations, Basic Concepts, Main Results. We consider quantum lattice systems on $\mathbb{Z}^{2}$. These systems are described in detail in chapters 2 and 7 of [29]. See also [30]. The $C^{*}$-algebra $\mathscr{A}$ associated with such systems has the following local structure: for each $x \in \mathbb{Z}^{2}, \mathscr{A}_{x}$ is a subalgebra of $\mathscr{A}$ which is isomorphic to the algebra of all bounded, linear operators $B(\mathbb{H})$ on a fixed, finite-dimensional Hilbert space $\mathbb{H}$. The subalgebras $\mathscr{A}_{x}$ and $\mathscr{A}_{y}$ commute elementwise, for $x \neq y$. Let $\Lambda$ be a subset of $\mathbb{Z}^{2}$. Then $\mathscr{A}(\Lambda)$ is the norm closure of the algebra generated by $\left\{\mathscr{A}_{x}: x \in \Lambda\right\}$. In particular $\mathscr{A}$ is the norm closure of $\mathscr{A}\left(\mathbb{Z}^{2}\right)$. A quantum lattice system is specified by prescribing an interaction potential. For simplicity we consider only two-body translation-invariant potentials, but our methods extend to more general 
interactions: with each two-element subset, $X$, of $\mathbb{Z}^{2}$ we associate a selfadjoint operator, $\phi(X)$, in $\mathscr{A}(X)$ such that

$$
\|\Phi\|=\sum_{X \ni 0}\|\phi(X)\|<\infty .
$$

The Hamiltonian operator, $U(\Lambda)$, which determines the dynamics of the system confined to a finite region $\Lambda$ of the lattice is defined by

$$
U(\Lambda)=\sum_{X \subset \Lambda} \phi(X)
$$

Under our assumptions on the interaction potential $\phi$, it is known that, for all $a \in \mathscr{A}$ and $t \in \mathbb{R}$,

$$
\alpha_{t}(a)=\lim _{\Lambda>\mathbb{Z}^{2}} e^{i t U(\Lambda)} a e^{-i t U(\Lambda)}
$$

exists and defines a strongly continuous one-parameter group of automorphisms of $\mathscr{A}$; see e.g. [29]. That group describes the time-evolution of the observables of the system in the thermodynamic limit.

Let $G$ be a compact, connected Lie group, and let $g \rightarrow V(g)$ be a unitary representation of $G$ on $\mathbb{H}$ which obviously determines a representation of $G$ as a group of automorphisms of $B(\mathbf{I H})$,

$$
a \rightarrow V(g) a V(g)^{*}, \quad a \in B(\mathbb{H}) .
$$

We suppose that the map

$$
g \rightarrow V(g) a V(g)^{*}, \quad a \in B(\mathbb{H}),
$$

is continuous for each $a \in B(\mathbb{H})$. (This is automatic when $\operatorname{dim} \mathbb{H}<\infty$.) Since $B(\mathbb{H}) \cong \mathscr{A}_{x}$, we obtain, for each $x \in \mathbb{Z}^{2}$, a strongly continuous representation of $G$ by automorphisms of $\mathscr{A}$, which we denote by $\gamma_{x}(g)$. Furthermore, we define

$$
\gamma(g)(a)=\left(\bigotimes_{x \in \Lambda} \gamma_{x}(g)\right)(a) .
$$

where $\Lambda$ is an arbitrary, finite subset of $\mathbb{Z}^{2}$, and $a \in \mathscr{A}(\Lambda)$. By continuity, this determines a representation $\gamma(\cdot)$ of $G$ as a group of automorphisms of $\mathscr{A}$. Let $\varphi$ be a state on $\mathscr{A}$. Then the state $\varphi_{g}, g \in G$, is defined by

$$
\varphi_{g}(a)=\varphi(\gamma(g)(a)), \quad a \in \mathscr{A} .
$$

A state $\varphi$ is $G$-invariant if $\varphi_{g}=\varphi, \forall g \in G$.

Theorem 2. In the situation described above, and if for all $X=\{x, y\} \subset \mathbb{Z}^{2}$

a) $\|\phi(X)\| \leqq C|x-y|^{-4}$ for some constant $C$,

b) $\gamma(g)(\phi(X))=\phi(X), \quad \forall g \in G$,

then all $\alpha_{t}$-KMS states of the system are G-invariant.

Remarks. 1) This result extends to more general potentials, $\phi$, with manybody interactions of "short range", (sufficiently rapid fall-off of $\|\phi(X)\|$ in $\operatorname{diam}(X)$, [30]). 
2) This result is best possible, since there exist two-dimensional systems with a continuous symmetry group $G$, and two-body interactions, $\phi$, with

$$
\|\phi(\{x, y\})\| \approx C|x-y|^{-4+\varepsilon},
$$

$\varepsilon>0$ arbitrarily small, for which spontaneous breaking of $G$ is known to occur, [31], [32].

3) The absence of spontaneous breaking of continuous symmetries in twodimensional systems with short range interactions does not exclude the existence of phase transitions (non-uniqueness of the Gibbs state) in such systems. An example of such a transition, in the classical case, has been constructed by Shlosman [33].

4) For mathematical background used in the proof of Theorem 2 we refer the reader to the work of Araki, [34], [35].

3.2. Proof of Theorem 2. Since each $\alpha_{t}$-KMS state has a unique decomposition as a direct integral of extremal $\alpha_{t}$-KMS states, it is sufficient to prove the theorem for extremal KMS states. Moreover, it is clearly sufficient to only consider closed one parameter subgroups $H$ of $G$. Since $G$ is compact $H \cong \mathbb{R} / \mathbb{Z}=S^{1}$. Furthermore, we consider only potentials, $\phi(X)$, such that

$$
\sum_{0 \neq x \in \mathbb{Z}^{2}}\|\phi(\{0, x\})\||x|^{2}<\infty .
$$

The general case is obtained as in [16]. Let $\varphi$ be an extremal KMS state (at inverse temperature $\beta=1$ ), and let $g$ be a fixed element of $H$. Since $H \cong S^{1}$, it can be parametrized by the numbers in $[0,1)$. Let

$$
\Lambda(L)=\left\{x=\left(x^{1}, x^{2}\right) \in \mathbb{Z}^{2}|| x^{1}|\leqq L, \quad| x^{2} \mid \leqq L\right\} .
$$

For each $L$, we are going to construct a family of states, $\psi_{L}$, on $\mathscr{A}$ such that

$$
\psi_{L}(a)=\varphi_{g}(a), \quad \forall a \in \mathscr{A}(\Lambda(L))
$$

and

$$
S\left(\psi_{L} \mid \varphi\right) \leqq K<\infty, \quad \text { uniformly in } L,
$$

where $S\left(\psi_{L} \mid \varphi\right)$ is the relative entropy of the two states defined by

$$
S\left(\psi_{L} \mid \varphi\right)=-\varphi\left(\log \Delta_{\psi_{L}, \varphi}\right)
$$

Here $\Delta_{\psi_{L}, \varphi}$ is the relative modular operator of the states $\psi_{L}$ and $\varphi$. For properties of $S(\cdot \mid \cdot)$ see $[36,18]$. Using the monotonicity property of $S(\cdot \mid \cdot)$, the fact that the restrictions of $\psi_{L}$ and $\varphi_{g}$ to $\mathscr{A}(\Lambda(L))$ coincide, and Lemmas 2 and 3 of [18], we obtain that $\varphi_{g}=\varphi$. The ideas of the proof of this last statement are the same as those in the classical case: From (3.3) we deduce that $\varphi$ and $\varphi_{g}$ are quasiequivalent (which corresponds to equivalence of measures in the abelian case), and therefore since $\varphi$ is extremal, $\varphi=\varphi_{g}$, as in the classical case.

Let $\Lambda(L)$ be fixed. Let $\tau_{+}$be the automorphism of $\mathscr{A}$ defined by

$$
\tau_{+}=\bigotimes_{x \in \Lambda(2 L)} \gamma_{x}\left(g_{x}\right)
$$


where

$$
\begin{gathered}
g_{x}=g, \quad x \in \Lambda(L), \\
g_{x}=\frac{L-k}{L} g, \quad x=\left(x^{1}, x^{2}\right), \max \left(\left|x^{1}\right|,\left|x^{2}\right|\right)=L+k .
\end{gathered}
$$

We define $\tau_{-}$by replacing $g$ by $-g$. We introduce

$$
\psi_{L}(a) \equiv \psi_{+}(a)=\varphi\left(\tau_{+}(a)\right), \quad a \in \mathscr{A}
$$

and

$$
\psi_{-}(a)=\varphi\left(\tau_{-}(a)\right), \quad a \in \mathscr{A}
$$

Let $\left(\mathrm{H}_{\varphi}, \Omega_{\varphi}, \pi_{\varphi}\right)$ be the GNS representation associated with the algebra $\mathscr{A}$ and the state $\varphi$. We also denote by $\varphi$ the extension of the state to the von Neumann algebra $\mathscr{M}=\pi_{\varphi}(\mathscr{A})^{\prime \prime}$. Since $\varphi$ is a KMS state on $\mathscr{A}$, its extension to $\mathscr{M}$ is a positive, faithful, normal state on $\mathscr{M}$, and the theory of relative hamiltonians holds [37]. Let

and

$$
h=\sum_{X \cap \Lambda(2 L) \neq \emptyset} \phi(X),
$$

$$
h_{ \pm}=\tau_{ \pm}^{-1}(h)
$$

Note that $h$ and $h_{ \pm}$are in $\mathscr{A}$.

We now consider local perturbations, $\varphi^{h}, h \in \mathscr{A}$, of the state $\varphi$ which (loosely speaking) are defined as follows: Let $\Delta \equiv e^{H}$ be the modular operator associated with $\varphi$. Here $-H$ is a renormalized version of the formal Hamilton operator $\lim _{\Lambda>\mathbb{Z}^{2}} U(\Lambda)$ on $\mathbb{H}_{\varphi}$. The perturbed state, $\varphi^{h}$, is defined by

$$
\varphi^{h}(a)=\left\langle e^{1 / 2(H+h)} \Omega_{\varphi}, \pi_{\varphi}(a) e^{1 / 2(H+h)} \Omega_{\varphi}\right\rangle .
$$

It is not very hard to verify that $\varphi^{h}$ satisfies the KMS condition for the automorphism group, $\alpha^{h}$, given by

$$
\alpha_{t}^{h}(a)=\lim _{\Lambda \rightarrow \mathbb{Z}^{2}} e^{i t(U(\Lambda)-h)} a e^{-i t(U(\Lambda)-h)} .
$$

Lemma 3.1. The states $\psi_{ \pm}$satisfy

$$
\psi_{ \pm}=\varphi^{h-h_{ \pm}} .
$$

Proof. Since $\varphi$ is KMS it satisfies the Gibbs condition of Araki [35]. Therefore the restriction of $\varphi^{h}, h=\sum_{X \cap \Lambda(2 L) \neq \emptyset} \phi(X)$, to $\mathscr{A}(\Lambda(2 L))$ is the normalized trace, and $\varphi^{h}$ is $\tau_{ \pm}$-invariant. We denote $\varphi^{h}$ by $\chi$. Let $\left(\mathbb{H}_{\chi}, \Omega_{\chi}, \pi_{\chi}\right)$ be the GNS representation of $\chi$. Let $H_{\chi}$ be the unique selfadjoint operator on $\mathbb{H}_{\chi}$ with

and

$$
H_{\chi} \Omega_{\chi}=0
$$

$$
\pi_{\chi}\left(\sigma_{t}^{\chi}(a)\right)=e^{i t H_{\chi}} \pi_{\chi}(a) e^{-i t H_{\chi}},
$$


where $\sigma_{t}^{\chi}(\cdot)$ is the modular automorphism group associated with $\chi$. Then

$$
\varphi(a)=\left\langle e^{1 / 2\left(H_{\chi}-h\right)} \Omega_{\chi}, \pi_{\chi}(a) e^{1 / 2\left(H_{\chi}-h\right)} \Omega_{\chi}\right\rangle,
$$

since $\varphi=\chi^{-h}$. Since $\chi$ is $\tau_{+}$-invariant there exists a unitary operator, $U_{+}$, on $\mathbb{H}_{\chi}$ such that

$$
U_{+} \pi_{\chi}(a) U_{+}^{*}=\pi_{\chi}\left(\tau_{+} a\right)
$$

and

$$
U_{+} \Omega_{\chi}=\Omega_{\chi}, \quad U_{+} e^{i t H_{\chi}}=e^{i t H_{\chi}} U_{+} .
$$

By using the Trotter product formula, an analytic continuation of (3.4) to imaginary time and

$$
U_{+}^{*} h U_{+}=\tau_{+}^{-1}(h)=h_{+},
$$

one sees that

$$
U_{+}^{*} e^{1 / 2\left(H_{x}-h\right)} U_{+}=e^{1 / 2\left(H_{x}-h_{+}\right)} .
$$

Therefore

$$
\begin{aligned}
\psi_{+}(a) & =\varphi\left(\tau_{+}(a)\right) \\
& =\left\langle e^{1 / 2\left(H_{\chi}-h\right)} \Omega_{\chi}, U_{+} \pi_{\chi}(a) U_{+}^{*} e^{1 / 2\left(H_{\chi}-h\right)} \Omega_{\chi}\right\rangle \\
& =\left\langle U_{+}^{*} e^{1 / 2\left(H_{\chi}-h\right)} \Omega_{\chi}, \pi_{\chi}(a) U_{+}^{*} e^{1 / 2\left(H_{\chi}-h\right)} \Omega_{\chi}\right\rangle \\
& =\left\langle e^{1 / 2\left(H_{\chi}-h_{+}\right)} \Omega_{\chi}, \pi_{\chi}(a) e^{1 / 2\left(H_{\chi}-h_{+}\right)} \Omega_{\chi}\right\rangle \\
& =\chi^{-h^{+}}(a)=\left(\varphi^{h}\right)^{-h^{+}}(a)=\varphi^{h-h^{+}}(a) .
\end{aligned}
$$

Next, we introduce again a duplication of the system and consider the two states $\varphi \otimes \varphi$ and $\psi_{+} \otimes \psi_{-}$. We compute $S\left(\psi_{+} \otimes \psi_{-} \mid \varphi \otimes \varphi\right)$. Since

$$
S\left(\psi_{+} \mid \varphi\right)=-\varphi\left(h-h_{+}\right),
$$

we have to bound terms like

$$
\varphi\left(\sum_{X \cap 1(2 L) \neq \emptyset}\left\{\tau_{+}^{-1} \phi(X)-\phi(X)\right\}\right)+\varphi\left(\sum_{X \cap 1(2 L) \neq \emptyset}\left\{\tau_{-}^{-1} \phi(X)-\phi(X)\right\}\right) .
$$

By the invariance of the potential the terms with $X \subset \Lambda(L)$ do not contribute to (3.5). Using the invariance of the potential

$$
\tau_{+}^{-1}(\phi(X))=\gamma_{y}\left(g_{x}-g_{y}\right)(\phi(X)),
$$

with $g_{x}=0$ if $x \notin \Lambda(2 L)$. Moreover we have

$$
\left|g_{x}-g_{y}\right| \leqq \frac{1}{L}|x-y|
$$

and $\left|g_{x}-g_{y}\right|=0$ if $x$ and $y$ are in $\Lambda(L)$. We expand the right-hand side of (3.6):

$$
\gamma_{y}\left(g_{x}-g_{y}\right)(\phi(X))=e^{i\left(g_{x}-g_{y}\right) A_{y}} \phi(X) e^{-i\left(g_{x}-g_{y}\right) A_{y}} .
$$


( $A_{y}$ is some selfadjoint element of $\mathscr{A}_{y}$.) We get

$$
\begin{aligned}
\gamma_{y}\left(g_{x}-g_{y}\right)(\phi(X))= & \phi(X) \\
& +i\left(g_{x}-g_{y}\right)\left[A_{y}, \phi(X)\right]+\frac{i^{2}}{2}\left(g_{x}-g_{y}\right)^{2}\left[A_{y},\left[A_{y}, \phi(X)\right]\right]+\ldots
\end{aligned}
$$

We do the same thing for $\tau_{-}^{-1}(\phi(X))$. Using the definition of $\tau_{+}$and $\tau_{-}$(3.1), (3.7) and (3.9) we see that only terms of order $O\left(\frac{1}{L^{p}}\right), p \geqq 2$, contribute to $S\left(\varphi_{+} \otimes \varphi_{-} \mid \varphi \otimes \varphi\right)$, that the second-order terms give a finite contribution, and that the higher order terms give a contribution of order $O\left(\frac{1}{L}\right)$. Therefore we have constructed a family of states $\psi_{L}$ satisfying (3.2) and (3.3). This ends the proof.

Remark. Our proof can be extended to certain quantum lattice systems where $\mathscr{A}_{x}$ is infinite dimensional, and $\phi(X)$ is not necessarily bounded.

We also believe that the results of Sect. 2 could be extended to quantummechanical particle systems with Fermi statistics by combining the methods of the present section with an extension of Ruelle's work [25] to quantum systems, but we have not checked this.

\section{Non-existence of Gibbs State for Two-Dimensional Harmonic and Anharmonic Crystal}

4.1. Introduction, Main Results. We consider unbounded spin systems on $\mathbb{Z}^{2}$. The state space of the spin, $s_{x}$, at $x \in \mathbb{Z}^{2}$ is the measure space $\left(\mathbb{R}, \mathbf{B}, d s_{x}\right)$, where $\mathbf{B}$ is the Borel $\sigma$-algebra, and $d s_{x}$ the Lebesgue measure. We use the following notations: If $\Lambda$ is a subset of $\mathbb{Z}^{2}, \mathbf{B}(\Lambda)=\prod_{x \in \Lambda} \mathbf{B}, s_{\Lambda}=\left(s_{x}: x \in \Lambda\right), d s_{\Lambda}=\prod_{x \in \Lambda} d s_{x}$ and $\bar{\Lambda}=\mathbb{Z}^{2} \backslash \Lambda$. In particular we set $\underline{s}=s_{\mathbb{Z}^{2}}, \stackrel{x \in A}{d \underline{s}}=\prod_{x \in \mathbb{Z}^{2}} d s_{x}$. We define $\mid x \in=\max \left\{\left|x^{1}\right|,\left|x^{2}\right|\right\}$, for $x=\left(x^{1}, x^{2}\right) \in \mathbb{Z}^{2}$. The configuration space of the system is the measure space $\left(\Omega=\prod_{x \in \mathbb{Z}^{2}} \mathbb{R}, \mathbf{B}\left(\mathbb{Z}^{2}\right), d \underline{s}\right)$. We consider only two-body interactions of the type

$$
\phi_{X}\left(s_{x}, s_{y}\right)=J(x-y) U\left(s_{x}-s_{y}\right),
$$

where $X=\{x, y\} \subset \mathbb{Z}^{2}$.

Assumption A. $U: \mathbb{R} \rightarrow \mathbb{R}$ is

a) twice continuously differentiable,

b) $U(s)=U(-s)$

c) There exists a natural number $p \geqq 0$ and a constant $K$ such that $\left|\frac{d^{2}}{d s^{2}} U(s)\right| \leqq K|s|^{p}$. 
Assumption B. There exists a constant $C$ such that

$$
\sum_{x \in \mathbb{Z}^{2}}|J(x)||x|^{2} \leqq C<\infty .
$$

Let $\Lambda$ be a bounded set. We introduce

$$
H_{\Lambda}\left(s_{\Lambda} \mid \hat{s}\right)=\sum_{X \cap \Lambda \neq \emptyset} \phi_{X}\left(s_{x}, s_{y}\right), \quad s_{y}=\hat{s}_{y} \quad \text { if } \quad y \notin \Lambda,
$$

and

$$
Z(\Lambda \mid \hat{s})=\int_{\mathbb{R}^{\Lambda}} d s_{\Lambda} \exp \left(-\beta H\left(s_{\Lambda} \mid \hat{s}\right)\right)
$$

We define the Gibbs distribution in $\Lambda$, given the boundary condition $\hat{s}$ in $\Omega$, as the probability measure on $\left(\mathbb{R}^{\Lambda}, \mathbf{B}(\Lambda)\right)$ whose Radon-Nikodym density with respect to $d s_{\Lambda}$ is

$$
P_{\Lambda}\left(s_{\Lambda} \mid \hat{s}\right)=Z(\Lambda \mid \hat{s})^{-1} \exp \left(-\beta H_{\Lambda}\left(s_{\Lambda} \mid \hat{s}\right)\right) .
$$

This expression is well-defined for all configurations $\hat{s}$ for which (4.1) is defined, for almost all $s_{\Lambda}$, and $0<Z(\Lambda \mid \hat{s})<\infty$. A probability measure $P$ on $\left(\Omega, \mathbf{B}\left(\mathbb{Z}^{2}\right)\right)$ is a $G i b b s$ state if for all bounded sets $\Lambda$ (4.2) is well-defined for $P$-almost all $\hat{s}$, and the conditional probability of $P$ with respect to $\boldsymbol{B}(\bar{\Lambda})$ is given by the Gibbs distribution in $\Lambda$.

Theorem 3. If Assumptions $A$ and $B$ are satisfied, then there is no Gibbs state P such that

$$
P\left(\left|s_{x}\right|^{q}\right) \leqq C_{q}, \quad q \geqq p,
$$

where $C_{q}$ is a constant independent of $x$, but arbitrarily large.

Remarks.

1) If $\max _{s \in \mathbb{R}}\left|\frac{d^{2}}{d s^{2}} U(s)\right| \leqq k \leqq \infty$, then there is no Gibbs state. In particular this is the case for the harmonic crystal, since $U(s)=s^{2}$. In the anharmonic case, $U(s)=s^{4}$, there is no Gibbs state with finite second moment.

2) In the class of models considered, the potential energy $U(\cdot)$ is $\mathbb{R}$-invariant. $G=\mathbb{R}$ is the internal symmetry group. This is an example of a non-compact, connected Lie group. It acts on the state space for a spin $(\mathbb{R}, \mathbf{B})$. It is possible to consider more general classes of $G$-invariant potentials, where $G$ is a non-compact, connected Lie group acting on a very general state space (see [20]).

There is another way to express the results, which makes a connection with [22]. Let us fix a boundary condition $\hat{s}$ and choose a sequence of bounded sets $\Lambda_{n}, \Lambda_{n} \uparrow \mathbb{Z}^{2}$, so that

$$
P_{\Lambda_{n}}\left(s_{\Lambda_{n}} \mid \hat{s}\right) d s_{\Lambda_{n}}
$$

are well-defined for all $n$. Let $\left\langle\left|s_{0}\right|^{q}\right\rangle_{n}$ be the expected value of $\left|s_{0}\right|^{q}$ with respect to the probability measure (4.3). For example we can take $U(s)=s^{2}$ or $s^{4}, \hat{s}_{x}=0$ for all $x \in \mathbb{Z}^{2}$, and $\Lambda_{n}=\{x:|x| \leqq n\}$. 
Theorem 4. If Assumptions $A$ and $B$ are satisfied for some $p>0$, then

$$
\sup _{x \in \mathbb{Z}^{2}} \liminf _{n \rightarrow \infty}\left\langle\left|s_{x}\right|^{q}\right\rangle_{n}=+\infty
$$

for $q \geqq p$. If $A$ is satisfied with $p=0$, then

$$
\liminf _{n \rightarrow \infty}\left\langle\left|s_{0}\right|^{q}\right\rangle_{n}=+\infty
$$

for all $q>0$. In particular, this holds for the harmonic crystal.

\subsection{Proofs of Theorems 3 and 4}

Proof of Theorem 3. Let $P$ be a Gibbs state such that

$$
\sup _{x \in \mathbb{Z}^{2}} P\left(\left|s_{x}\right|^{p}\right) \leqq C<\infty \text {. }
$$

We define for all $L \in \mathbb{N}$

$$
F(L)=\sum_{k=1}^{L} \frac{1}{k}
$$

Let $t \in \mathbb{R}$ be fixed and

$$
a_{x}^{L}(t)= \begin{cases}t, & \text { if } x=0 \\ t F^{-1}(L) \sum_{k=|x|}^{L} \frac{1}{k}, & \text { if } 1 \leqq|x| \leqq L \\ 0, & \text { if }|x| \geqq L .\end{cases}
$$

We define a transformation $T_{t}$ on $\Omega$ (depending on $L$ ) by setting

$$
\left(T_{t} \underline{s}\right)_{x}=s_{x}+a_{x}^{L}(t) .
$$

Let $P_{t}$ be the probability defined by

$$
P_{t}(f)=T_{t} P(f)=\int P(d s) f\left(T_{t}^{-1} s\right)
$$

Lemma 4.1. $P_{t}$ is absolutely continuous with respect to $P$ and

$$
\frac{d P_{t}}{d P}(\underline{s})=\exp \left(-\beta \sum_{X \cap A_{L} \neq \emptyset}\left(\phi_{X}\left(T_{t} s_{x}, T_{t} s_{y}\right)-\phi_{X}\left(s_{x}, s_{y}\right)\right)\right)
$$

with

$$
\Lambda_{L}=\left\{x \in \mathbb{Z}^{2}:|x| \leqq L\right\} .
$$

Proof. This follows from the fact that $P$ is a Gibbs state; see Eq. (4.2).

We now introduce a duplicate system and consider the probability measures $P \otimes P$ and $P_{t} \otimes P_{-t}$ on $\Omega \times \Omega$ where $P_{-t}=T_{-t} P$. The relative entropy is given by

$$
S\left(P_{t} \otimes P_{-t} \mid P \otimes P\right)=-\int P(d \underline{s}) P\left(d \underline{s}^{\prime}\right) \log \frac{d\left(P_{t} \otimes P_{-t}\right)}{d(P \otimes P)}\left(\underline{s}, \underline{s}^{\prime}\right) .
$$


Lemma 4.2. Let $P$ be a Gibbs state such that (4.4) holds. Then one can choose $L=L(t)$ in (4.6) such that

$$
0 \leqq S\left(P_{t} \otimes P_{-t} \mid P \otimes P\right) \leqq 1 .
$$

Proof. The proof is like that of Lemma 2.2. Let $X=\{x, y\}$.

$$
\begin{aligned}
\phi_{X}\left(T_{t} s_{x}, T_{t} s_{y}\right)= & \phi_{X}\left(s_{x}+a_{x}^{L}(t), s_{y}+a_{y}^{L}(t)\right) \\
= & J(x-y) U\left(s_{x}-s_{y}+a_{x}^{L}(t)-a_{y}^{L}(t)\right) \\
= & J(x-y)\left[U\left(s_{x}-s_{y}\right)+\frac{d}{d s} U\left(s_{x}-s_{y}\right)\left(a_{x}^{L}(t)-a_{y}^{L}(t)\right)\right. \\
& \left.+\frac{1}{2} \frac{d^{2}}{d s^{2}} U\left(s_{x}-s_{y}+\theta\right)\left(a_{x}^{L}(t)-a_{y}^{L}(t)\right)^{2}\right] .
\end{aligned}
$$

In formula (4.7) $|\theta| \leqq|t|$. Using (4.4) we get

$$
\int P(d \underline{s})\left|\frac{d^{2}}{d s^{2}} U\left(s_{x}-s_{y}+\theta\right)\right| \leqq K \int P(d \underline{s})\left|s_{x}-s_{y}+\theta\right|^{p} \leqq \sum_{m=0}^{p} C_{m}|t|^{m} \equiv Q(|t|),
$$

where $C_{m}$ are constants independent of $x$ and $y$. Therefore $S\left(P_{t} \otimes P_{-t} \mid P \otimes P\right)$ is bounded by

$$
\begin{aligned}
& \sum_{\{x, y\} \cap A_{L} \neq \emptyset}|J(x-y)|\left(a_{x}^{L}(t)-a_{y}^{L}(t)\right)^{2} Q(|t|) \\
& \quad=Q(|t|) \sum_{k=1}^{L} \sum_{x:|x|=k} \sum_{y:|y|>|x|}|J(x-y)|\left(a_{x}^{L}(t)-a_{y}^{L}(t)\right)^{2} .
\end{aligned}
$$

For $|y|>|x|$ we have that

$$
\left|a_{x}^{L}(t)-a_{y}^{L}(t)\right| \leqq \frac{|x-y|}{|x|} \cdot \frac{t}{F(L)} .
$$

Thus (4.8) is smaller than

$$
\begin{aligned}
& Q(|t|) t^{2} \frac{1}{(F(L))^{2}} \sum_{k=1}^{L} \sum_{x:|x|=k} \frac{C}{|x|^{2}} \\
& =Q(|t|) t^{2} \frac{C}{(F(L))^{2}} \sum_{k=1}^{L} \frac{8}{k}=Q(|t|) t^{2} \frac{8 \cdot C}{F(L)} .
\end{aligned}
$$

Therefore, for any fixed $t$, we can find $L=L(t)$ so that (4.9) is smaller than one.

Let $v$ be the probability measure which is the projection on the state space of the spin at $x=0$ of the Gibbs state $P$. Likewise, let $v_{t}$ be the projection of $P_{t}$ on this state space. If $h$ is a measurable function on $\mathbb{R}$, then

$$
\int h(s) v_{t}(d s)=\int h(s-t) v(d s)
$$

Let $A \subset \mathbb{R}$ and $A^{c}=\mathbb{R} \backslash A$. Using Jensen's inequality and Lemma 4.2 we get

$$
0 \leqq-\left(v(A) \log \frac{v_{t}(A)}{v(A)}+v\left(A^{c}\right) \log \frac{v_{t}\left(A^{c}\right)}{v\left(A^{c}\right)}\right) \leqq 1,
$$


uniformly in $t$. Let I be a finite interval of $\mathbb{R}$ such that $v(I)=\delta>0$. Then $v_{t}(I) \geqq \varepsilon(\delta)$ $>0$ uniformly in $t$. But then (4.10) is incompatible with the fact that $v$ is a probability measure.

Proof of Theorem 4. Let us suppose first that Assumption A is satisfied with $p>0$, and assume temporarily that

$$
\sup _{x \in \mathbb{Z}^{2}} \lim _{n} \inf \left\langle\left|s_{x}\right|^{p}\right\rangle_{n} \leqq C<\infty
$$

Then we can find a subsequence of bounded sets, denoted again by $\Lambda_{n}$, such that

$$
\left\langle\left|s_{x}\right|^{p}\right\rangle_{n} \leqq C<\infty,
$$

with $C$ independent of $n$ and $x$. Let $t$ be fixed. We can prove Lemma 4.2 with $P$ replaced by $\langle\cdot\rangle_{n}$ if $n$ is large enough. Let $\mu_{n}$ be the projection of $\langle\cdot\rangle_{n}$ on the state space of the spin at $x=0$. Let $A \subset \mathbb{R}, A^{c}=\mathbb{R} \backslash A, A_{t}=\{x: x+t \in A\}$. For $n$ large enough

$$
0 \leqq-\left(\mu_{n}(A) \log \frac{\mu_{n}\left(A_{t}\right)}{\mu_{n}(A)}+\mu_{n}\left(A^{c}\right) \log \frac{\mu_{n}\left(A_{t}^{c}\right)}{\mu_{n}\left(A^{c}\right)}\right) \leqq 1,
$$

by Lemma 4.2 and Jensen's inequality, as above. Since $p>0$ and (4.11) holds, we obtain, using Tschebyscheff's inequality,

$$
\mu_{n}(\{x:|x| \geqq \lambda\}) \leqq \frac{C}{\lambda^{p}}
$$

Using (4.12) with $A=\{x:|x| \leqq \lambda\}$ we can find an $\varepsilon>0$ so that, for $\lambda \geqq \lambda_{0}$ and $n$ large enough,

$$
\mu_{n}\left(A_{t}\right) \geqq \varepsilon .
$$

Now we choose $t=2 \lambda$ and get a contradiction with (4.13) if $\lambda$ and $n$ are large enough.

The case $p=0$ is simpler, since we can prove Lemma 4.2 for any sequence $\Lambda_{n}$. Then we repeat the above proof with $q>p$ instead of $p$.

Remark. It is an interesting open problem to prove that

$$
\left\langle\left|s_{x}\right|^{2}\right\rangle_{n} \leqq \text { const }<\infty,
$$

uniformly in $n$, for the anharmonic crystal, with $U$ satisfying Assumption $A$ with $p$ $>0$ and $J$ nearest neighbor, in $d \geqq 3$ dimensions; see also [22].

Acknowledgements. C.P. would like to thank Prof. J. Lascoux and the members of Centre de Physique Théorique, Ecole Polytechnique, for very kind hospitality. He thanks O. Besson for several useful discussions on Sect. 3. We thank J. Lebowitz for stimulating remarks.

\section{References}

1. Mermin, N. D., Wagner, H.: Phys. Rev. Lett. 17, 1133 (1966)

2. Mermin, N. D.: J. Math. Phys. 8, 1061 (1967)

3. Mermin, N. D.: Phys. Rev. 176, 250 (1968) 
4. Ezawa, H., Swieca, J.: Commun. Math. Phys. 5, 330 (1967)

5. Coleman, S.: Commun. Math. Phys. 31, 259 (1978)

6. Mermin, N. D.: J. Phys. Soc. Jpn 26 Suppl., 203 (1969)

7. Hohenberg, P. C.: Phys. Rev. 158, 383 (1967)

8. Bouziane, M., Martin, P.A.: J. Math. Phys. 17, 1848 (1976)

9. Jasnow, D., Fisher, M. E.: Phys. Rev. B 3, 895 and 907 (1971)

10. McBryan, O.A., Spencer, T.: Commun. Math. Phys. 53, 299 (1977)

11. Shlosman, S. B.: Teor. Mat. Fiz. 37, 1118 (1978)

12. Vuillermot, P.A., Romerio, M. V.: Commun. Math. Phys. 41, 281 (1975)

13. Garrison, J. C., Wong, J., Morrison, H. L.: J. Math. Phys. 13, 1735 (1972). See also Klein, A., Landau, L.J., Shucker, D. S.: Preprint (1981)

14. Dobrushin, R. L., Shlosman, S. B.: Commun. Math. Phys. 42, 31 (1975)

15. Shlosman, S. B.: Teor. Mat. Fiz. 33, 86 (1977)

16. Pfister, C. E.: Commun. Math. Phys. 79, 181 (1981)

17. Simon, B., Sokal, A. D.: J. Stat. Phys. (in press)

18. Araki, H.: Commun. Math. Phys. 44, 1 (1975)

19. Herring, C., Kittel, C.: Phys. Rev. 81, 869 (1951); see footnote 8a, p. 873

20. Dobrushin, R. L., Shlosman, S. B.: in "Multicomponent Random Systems", ed. by Dobrushin, R. L., Sinai, Y.G.: Advances in probability and related topics, Vol. 6, New York, Basel: Marcel Dekker, Inc., 1980

21. Jona Lasinio, G., Pierini, S., Vulpiani A.: Preprint 1980

22. Brascamp, H.J., Lieb, E.H., Lebowitz, J.L.: Proceedings of 40th session of the International Statistical Institute, Warszawa (1975)

23. Dobrushin, R. L.: Teor. Mat. Phys. 4, 101 (1970)

24. Georgii, H. O.: Canonical Gibbs Measures, Lecture Notes in Mathematics 760, Berlin-HeidelbergNew York: Springer-Verlag 1979

25. Ruelle, D.: Commun. Math. Phys. 18, 127 (1970)

26. Nguyen, X.X., Zessin, H.: Math. Nachr. 88, 105 (1979)

27. Föllmer, H.: in Séminaire de Probabilitiés IX, Lecture Notes in Mathematics 465, p. 305, BerlinHeidelberg-New York: Springer-Verlag 1975

28. Gruber, Ch., Martin, P.A.: Phys. Rev. Letters 45, 853 (1980) and Ann. Phys. 131, 56 (1981)

29. Ruelle, D.: Statistical mechanics. New York: W.A. Benjamin Inc. 1969

30. Israel, R. B.: Convexity in the theory of lattice gases, Princeton, NJ: Princeton University Press 1979

31. Kunz, H., Pfister, C. E.: Commun. Math. Phys. 46, 245 (1976)

32. Fröhlich, J., Israel, R., Lieb, E. H., Simon, B.: Commun. Math. Phys. 62, 1 (1978)

33. Shlosman, S. B.: Commun. Math. Phys. 71, 207 (1980)

34. Araki, H.: in Colloques Internationaux C.N.R.S. $\mathrm{N}^{\circ}$ 248, 61, Editions du C.N.R.S., Paris, 1976

35. Araki, H.: in: $C^{*}$-algebras and their applications to statistical mechanics and quantum field theory. (ed. Kastler, D.), Amsterdam: North Holland 1976

36. Araki, H.: Publ. R.I.M.S. 11, 809 (1976)

37. Araki, H.: Publ. R.I.M.S. 9, 165 (1973)

Communicated by A. Jaffe

Received June 1, 1981 